\title{
On the Convergence of the WKB Series for the Angular Momentum Operator
}

\author{
Luca Salasnich $\bigoplus^{\dagger, \ddagger, \S}$ and Fabio Sattin $]^{, \boldsymbol{\top}, £}$ \\ †Dipartimento di Matematica Pura ed Applicata, Università di Padova, \\ Via Belzoni 7, 35131 Padova, Italy \\ ${ }^{\ddagger}$ Istituto Nazionale di Fisica Nucleare, Sezione di Padova, \\ Via Marzolo 8, 35131 Padova, Italy \\ $\S$ Istituto Nazionale di Fisica della Materia, Unità di Milano, \\ Via Celoria 16, 20133 Milano, Italy \\ ฯDipartimento di Ingegneria Elettrica, Università di Padova, \\ Via Gradenigo 6/a, 35131 Padova, Italy \\ $£$ Istituto Nazionale di Fisica della Materia, Unità di Padova, \\ Corso Stati Uniti 4, 35127 Padova, Italy
}

\begin{abstract}
In this paper we prove a recent conjecture [Robnik M and Salasnich L 1997 J. Phys. A: Math. Gen. 30 1719] about the convergence of the WKB series for the angular momentum operator. We demonstrate that the WKB algorithm for the angular momentum gives the exact quantization formula if all orders are summed. Finally, we discuss the supersymmetric semiclassical quantum mechanics (SWKB), which gives the correct quantization of the angular momentum at the leading order.
\end{abstract}

PACS: 03.65.-w , 03.65.Sq , 03.65.Ge

\footnotetext{
${ }^{1} \mathrm{E}-$ mail: salasnich@math.unipd.it

${ }^{2}$ Present address: Istituto Gas Ionizzati del C.N.R., Corso Stati Uniti 4, 35127 Padova, Italy. E-mail: sattin@igi.pd.cnr.it
} 


\section{Introduction}

The semiclassical methods used to solve the Schrödinger problem are of extreme importance to understand the global behaviour of eigenfunctions and energy spectra, since they allow to obtain analytic expressions. The leading semiclassical approximation (torus quantization) is just the first term of a certain $\hbar$-expansion, which is called WKB (Maslov and Fedoriuk 1981).

Recently it was observed (Prosen and Robnik 1993, Graffi, Manfredi and Salasnich 1994, Robnik and Salasnich 1997a-in the following this work will be referred to as I) that the torus quantization generally fails to predict the individual energy levels (and the eigenstates) within a vanishing fraction of the mean-energy level spacing. This conclusion is believed to be correct for general systems, including the chaotic ones. Therefore, a systematic study of the accuracy of semiclassical approximation is very important, especially in the context of quantum chaos (Casati and Chirikov 1995, Gutzwiller 1990). Since this is a difficult task, it has been attempted for simple systems, where in a few cases even exact solutions may be worked out (Dunham 1932, Bender, Olaussen and Wang 1977, Voros 1993, Robnik and Salasnich 1997a).

Robnik and Salasnich (1997b) (this work will be referred to as II) dealt with the WKB expansion for the Kepler problem: it was proved that an exact result is obtained once all terms are summed. In particular, the torus quantization (the leading WKB term) of the full problem is exact, even if the individual torus quantization of the angular momentum and of the radial Kepler problem separately are not, because the quantum corrections (i.e. terms higher than the torus quantization) compensate mutually term by term. In the paper II Robnik and Salasnich had to do a conjecture about the higher terms of the WKB expansion. This conjecture is perfectly reasonable but not rigorously proved. In this work our goal is to prove that the same result of II can be reached rigorously by means of a slightly modified procedure.

In the framework of the supersymmetric semiclassical quantization (SWKB), 
Comtet, Bandrauk and Campbell (1985) obtained at the leading order the exact quantization of the radial part of the Kepler problem by using the correct value $L^{2}=\hbar^{2} l(l+1)$. In the last section we complete the result of Comtet, Bandrauk and Campbell (1985). In fact, we show that also the exact quantization of the angular momentum is obtained at the first order of the SWKB expansion.

\section{Eigenvalue problem for the angular momen- tum}

The eigenvalue equation of the angular momentum operator (Landau and Lifshitz 1977) is

$$
\hat{L}^{2} Y(\theta, \phi)=\lambda^{2} \hbar^{2} Y(\theta, \phi)
$$

with

$$
\hat{L}^{2}=\hat{P}_{\theta}^{2}+\frac{\hat{P}_{\phi}^{2}}{\sin ^{2}(\theta)}=-\hbar^{2}\left(\frac{\partial^{2}}{\partial \theta^{2}}+\cot (\theta) \frac{\partial}{\partial \theta}\right)-\hbar^{2} \frac{1}{\sin ^{2}(\theta)} \frac{\partial^{2}}{\partial \phi^{2}}
$$

After the substitution

$$
Y(\theta, \phi)=T(\theta) e^{i m \phi}
$$

we obtain

$$
T^{\prime \prime}(\theta)+\cot (\theta) T^{\prime}(\theta)+\left(\lambda^{2}-\frac{m^{2}}{\sin ^{2}(\theta)}\right) T(\theta)=0 .
$$

We shall consider the azimuthal quantum number $m$ as fixed. As well known, Eq. (4) is exactly solvable. Its eigenvalues and eigenfunctions are known from any text of quantum mechanics (see, e.g. Landau and Lifshitz 1977): the former are $\lambda^{2}=l(l+1), l \geq m$; the latter are the associate Legendre polynomials.

The WKB expansion for Eq. (4) has been studied in II; it was shown that higher-order terms quickly increase in complexity. The method of solution 
is to find an analytical recursive expression for all the higher-order terms, to sum the entire infinite series, and show that it is convergent to the exact result. Instead of the original function $T$ we shall use the associated function $F$ :

$$
T(\theta)=\frac{F(\theta)}{\sqrt{\sin (\theta)}}
$$

from which we obtain

$$
F^{\prime \prime}(\theta)+\left[\left(\lambda^{2}+\frac{1}{4}\right)+\frac{1}{\sin ^{2}(\theta)}\left(\frac{1}{4}-m^{2}\right)\right] F(\theta)=0 .
$$

This equation has the standard form of the one-dimensional Schrödinger equation with $\hbar=2 M \equiv 1$. Its eigenvalues are $\left(\lambda^{2}+1 / 4\right)$. We make the substitution of variable: $x=\theta+\pi / 2$, and the positions $U=m^{2}-1 / 4, E=$ $\lambda^{2}+1 / 4$. Then Eq. (6) becomes

$$
-F^{\prime \prime}(x)+\frac{U}{\cos ^{2}(x)} F(x)=E F(x) .
$$

This is the main result of our paper, because the problem of the WKB quantization of Eq. (7) has already been dealt with in I. As we shall show, from I one proves that: i) Eq. (7) can be solved exactly ; ii) a semiclassical expansion of (7) may be carried on to all orders (i.e. all terms may be exactly and analytically computed and summed); iii) the exact and the semiclassical eigenvalues are the same.

\section{WKB series for the angular momentum}

We observe that in Eq. (7) does not appear $\hbar$, therefore an expansion in powers of this parameter is not possible. To override this difficulty a small parameter $\epsilon$ is introduced:

$$
-\epsilon^{2} F^{\prime \prime}(x)+\frac{U}{\cos ^{2}(x)} F(x)=E F(x) .
$$


This parameter $\epsilon$, which will be set to 1 at the end of the calculation, has formally the same role of $\hbar$ as ordering parameter. It has already been used in II to deal with the WKB expansion of (4). The formal WKB expansion for $F$ reads:

$$
F(x)=\exp \left(\frac{i}{\epsilon} \sum_{n=0}^{\infty} \sigma_{n}(x) \epsilon^{n}\right),
$$

and we obtain a recursion relation for the phases:

$$
\begin{gathered}
\left(\sigma_{0}^{\prime}(x)\right)^{2}=E-\frac{U}{\cos ^{2}(x)}, \\
\sum_{k=0}^{n} \sigma_{k}^{\prime}(x) \sigma_{n-k}^{\prime}(x)+\sigma_{n-1}^{\prime \prime}(x)=0, \quad n>0 .
\end{gathered}
$$

The quantization condition is obtained by requiring that the wavefunction be single valued:

$$
\oint d \sigma=\sum_{k=0}^{\infty} \oint d \sigma_{k}=2 \pi n_{\theta},
$$

where $n_{\theta}$ is an integer number. All odd terms higher than the first vanish when integrated along the closed contour since they are exact differentials (Bender, Olaussen and Wang 1977)

$$
\oint d \sigma_{2 k+1}=0, \quad k>0 .
$$

It may be proved by induction (see I) that the solution of (10-11) is

$$
\sigma_{n}^{\prime}(x)=\left(\sigma_{0}^{\prime}\right)^{1-3 n} P_{n}(\cos (x)) \sin ^{f(n)}(x),
$$

with $f(n)=0$ for $n$ even, $f(n)=1$ for $n$ odd,

$$
P_{n}(\cos (x))=\sum_{l=0}^{g(n)} C_{n, l} \cos ^{2 l-3 n}(x),
$$

with $g(n)=(3 n-2) / 2$ for $n$ even, $g(n)=(3 n-3) / 2$ for $n$ odd, $C_{0,0}=$ $1, C_{1,0}=U / 2, C_{2 k, 0}=(-1)^{k}(U / 2)^{2 k}\left(\begin{array}{l}1 / 2 \\ k\end{array}\right)$, and $C_{2 k+1,0}=0, k>0$. It is not necessary to know the value of the other coefficients since one finds that all 
the terms proportional to $C_{n, l}, l>0$ disappear after integration. The integral (12) becomes (see I for more details)

$$
\begin{aligned}
\oint d \sigma & =\oint d \sigma_{0}+\oint d \sigma_{1}+\sum_{k>0}^{\infty} \oint d \sigma_{2 k} \\
& =2 \pi(\sqrt{E}-\sqrt{U})-\pi-\sum_{k>0}^{\infty} \frac{1}{2}\left(\left(\begin{array}{l}
\frac{1}{2} \\
k
\end{array}\right)\right) \frac{2 \pi}{(\sqrt{4 U})^{2 k-1}} \\
& =2 \pi\left(\sqrt{E}-\frac{1}{2}-\sum_{k=0}^{\infty} \frac{1}{2}\left(\left(\begin{array}{l}
\frac{1}{2} \\
k
\end{array}\right)\right) \frac{2 \pi}{(\sqrt{4 U})^{2 k-1}}\right)=2 \pi n_{\theta}
\end{aligned}
$$

but $\sum_{k=0}^{\infty}\left(\left(\begin{array}{l}\frac{1}{2} \\ k\end{array}\right)\right) x^{1-2 k}=\sqrt{1+x^{2}}$ for $|x|>1$, therefore Eq. (18) reads

$$
\sqrt{E}-\frac{1}{2} \sqrt{1+4 U^{2}}-\frac{1}{2}=\sqrt{\lambda^{2}+\frac{1}{4}}-\frac{1}{2} \sqrt{4 m^{2}}-\frac{1}{2}=n_{\theta} .
$$

Now, because $E=\lambda^{2}+1 / 4$ and $U=m^{2}-1 / 4$, we obtain

$$
\lambda^{2}=\left(m+n_{\theta}+\frac{1}{2}\right)^{2}-\frac{1}{4}=\left(m+n_{\theta}\right)\left(m+n_{\theta}+1\right),
$$

and, with the position $l=n_{\theta}+m$, we have

$$
\lambda^{2}=l(l+1)
$$

which is the expected result. Please note that the WKB series is convergent for $|x|>1$, thus for $m>0$.

We observe that the $\epsilon-$ expansion is equivalent to the $1 / U$-expansion (this is clear from the structure of Eq. 8). In the limit $U \rightarrow \infty$ it is easy to get the WKB expansion to the first order, which gives $\lambda^{2}=(l+1 / 2)^{2}$, i.e. the torus quantization of the angular momentum (Langer 1937).

\section{SWKB quantization of the angular momen- tum}

To perform the supersymmetric semiclassical quantization (SWKB) of Eq. (4) or (6), it is necessary to know the ground state wave-function $T_{0}(\theta)=$ 
$\sin ^{m}(\theta)$ and its eigenvalue $\lambda_{0}=m(m+1)$. Then we can define the supersymmetric (SUSY) potential

$$
\Phi(\theta)=-\frac{d \ln \left(F_{0}(\theta)\right)}{d \theta}=-\left(m+\frac{1}{2}\right) \cot (\theta),
$$

with

$$
F_{0}(\theta)=T_{0}(\theta) \sqrt{\sin (\theta)}
$$

From $\Phi$ the two SUSY partner potentials and Hamiltonians may be defined

$$
\begin{gathered}
H_{ \pm}=-\frac{d^{2}}{d \theta^{2}}+V_{ \pm}(\theta), \\
V_{ \pm}(\theta)=\Phi^{2}(\theta) \pm \Phi^{\prime}(\theta) .
\end{gathered}
$$

It is possible to prove (see Junker 1996 and references therein for details) that: i) the ground-state energy of $H_{-}, E_{-}^{0}$, vanishes; ii) all other eigenvalues of $H_{-}, E_{-}$, coincide with that of $H_{+}$; iii) the spectrum of $H_{-}$and that of (7) differ by a constant:

$$
E_{-}=\lambda^{2}+\frac{1}{4}-\left(\lambda_{0}^{2}+\frac{1}{4}\right)
$$

where $\lambda_{0}=m(m+1)$ is the eigenvalue of the ground state of Eq. (4).

Now we apply the SWKB formalism to $H_{-}$of Eq. (24). At the leading order one gets

$$
\int_{a}^{b} \sqrt{E_{-}-\Phi^{2}(x)} d x=n_{\theta} \pi
$$

with $a, b$ roots of

$$
E_{-}-\Phi^{2}(x)=0
$$

This formula is also referred to as CBC formula, from Comtet, Bandrauk and Campbell (1985). We observe that on the left hand side of the previous formulas $\Phi^{2}$ appears instead of the full potential $V_{-}$.

From Eqns. (22), (27) and (28) one easily finds

$$
\sqrt{E_{-}+\left(m+\frac{1}{2}\right)^{2}}-\left(m+\frac{1}{2}\right)=n_{\theta},
$$


with $b=-a=\arctan \sqrt{\frac{\left(m+\frac{1}{2}\right)}{E_{-}}}$. By inverting the previous formula we have

$$
E_{-}=\left(n_{\theta}+m+\frac{1}{2}\right)^{2}-\left(m+\frac{1}{2}\right)^{2},
$$

and, by using Eq. (26) with $\lambda_{0}=m(m+1)$, we get

$$
\lambda^{2}=\left(n_{\theta}+m\right)\left(n_{\theta}+m+1\right)
$$

which yields the exact quantization, after the position $l=n_{\theta}+m$.

\section{Conclusions}

The three-dimensional central potentials are fundamental in physics, and also the semiclassical treatment of them has implications in many fields: factorization properties of the one-dimensional potentials (Infeld and Hull 1957), general properties of the semiclassical quantization of the systems with more than one degree of freedom, both integrable or not. Nevertheless, until the paper of Robnik and Salasnich (1997b), no detailed study had been done on half of the problem, the WKB quantization of the angular part. Our present paper completes that work because it gives a rigorous proof of the convergence of the WKB series to the exact result. Moreover, in the last section, we have demonstrated that, by using SUSY quantum mechanics, the eigenvalue problem of the angular momentum operator can be solved exactly at the lowest order within the semiclassical approximation.

LS thanks Marko Robnik for many enlightening discussions. FS has been supported during this work by a grant of the Italian MURST. 


\section{References}

Barclay D T and Maxwell C J 1991 Phys. Lett. A 157357

Bender C M, Olaussen K and Wang P S 1977 Phys. Rev. D 161740

Casati G and Chirikov B V 1995 Quantum Chaos (Cambridge: Cambridge University Press)

Comtet A, Bandrauk A D, and Campbell D K 1985 Phys. Lett. B 150159

Dutt R, Khare A, and Sukhatme U P 1986 Phys. Lett. B 181295

Dunham J L 1932 Phys. Rev. 41713

Graffi S, Manfredi V R, Salasnich L 1994 Nuovo Cimento B 1091147

Gutzwiller M C 1990 Chaos in Classical and Quantum Mechanics (New York: Springer)

Infeld L and Hull T H 1957 Rev. Mod. Phys. 2321

Junker G 1996 Supersymmetric Methods in Quantum and Statistical Physics (Springer)

Landau L D and Lifshitz E M 1977 Quantum Mechanics (3rd Edition, Pergamon)

Langer R I 1937 Phys. Rev. 51669

Maslov V P and Fedoriuk M V 1981 Semi-Classical Approximations in Quantum Mechanics (Reidel Publishing Company)

Prosen T and Robnik M 1993 J. Phys. A: Math. Gen. 26 L37

Robnik M and Salasnich L 1997a J. Phys. A: Math. Gen. 301711

Robnik M and Salasnich L 1997b J. Phys. A: Math. Gen. 301719 